\title{
Research of dynamic properties of control systems with increased potential of robust stability in a class of two- parameter structurally stable maps by Lyapunov function.
}

\author{
Beisenbi M.A. ${ }^{1}$, Abdrakhmanova L.G. ${ }^{2}$ \\ ${ }^{1}$ Department of Automation and control of L.N. Gumilev Eurasian National University, Astana, Kazakhstan \\ ${ }^{2}$ Department of Automation and control of L.N. Gumilev Eurasian National University, Astana, Kazakhstan \\ beisenbi@mail.ru, leila2186@mail.ru
}

\begin{abstract}
The proposed paper presents a method of constructing a control system with increased potential of robust stability for systems with uncertain parameters in the two-parameter class of structurally stable maps of catastrophe theory to synthesize highly efficient management system, have the property of stability for any changes in uncertain parameters.

In conditions of parametric uncertainty increased potential of robust stability is a key factor in ensuring the protection of the control system from falling into the chaotic motion, and also guarantees the reliability of the system.

Research of robust stability control system based on the method of vector function Lyapunov, based on the geometric interpretation of the direct method A.M. Lyapunov, and the Morse lemma of catastrophe theory.

Keywords - control system, parameter uncertainty, robust stability, structurally stable maps, stability region.
\end{abstract}

\section{Introduction}

It is generally recognized that the majority of real control systems operate under some degree of uncertainty. In these cases, the uncertainty may be due to incomplete knowledge of parameters of the controlled plant or due to unpredictable time-varying characteristics in the process of operation. Therefore, the problem of robust stability is one of the most prominent problems of control theory and it is of great practical interest. In general, statement of the problem includes definition of the limits on parameter variations of the system that would be required to preserve stability. Clearly, these limits are determined by the region of stability due to parameter uncertainty of the plant and by controller settings.

Most of the known approaches [1] to implementing controls for systems with uncertain parameters rely on robust stability for the system with a given structure and linear control laws, or inertia less nonlinear (relay) characteristics. These methods do not allow designing a control system with expanded range of robust stability when uncertainty of the control parameters of the object and parameter drift are present.

There are currently no provisions for scientific research and development of control systems with a wide range of robust stability.

The results reported in [2] focus on dynamic systems and address the development of self-organizing processes of physical-chemical and biological systems. Models of these systems are established in the form of structurally stable maps using the catastrophe theory $[3,4]$ and studied as a universal model of evolution and self-organization in nature. It is therefore of particular interest, in terms of great uncertainty, to build a system of automatic control in the class of structurally stable maps associated with mathematical models of complex behavior of the system $[5,6]$, and it is consistent with many sustainable solutions.

This paper is devoted to the issues of synthesis of systems with robust stability for dynamic objects with uncertain parameters and construction of control systems in a class of two-parameter structurally stable maps $[5,6]$, which allows increasing the range of robust stability and improving quality characteristics of the control system.

The concept of building control systems with a high potential for robust stability of dynamic objects is based on the catastrophe theory $[3,4]$, where the main result are obtained in the form of basic structurally stable maps. They are limited and directly related to the number of control parameters.

Let the control system be described by the following state equation

$$
\dot{x}=A x+b u, y=c x, x \in R^{n}, y \in R .
$$

Control law is given in the form of two-parameter structurally stable maps $[1,2]$ :

$$
u_{i}=-x_{i}^{4}-k_{1 i} x_{i}^{2}+k_{2 i} x_{i}, i=1, \ldots, n
$$

A system given by (1) and (2) can be expanded as follows:

$$
\left\{\begin{array}{l}
\frac{d x_{1}}{d t}=x_{2}, \\
\frac{d x_{2}}{d t}=x_{3}, \\
\ldots \ldots \ldots \ldots \ldots \ldots, \\
\frac{d x_{n}}{d t}=-x_{1}^{4}+k_{1}^{\prime} x_{1}^{2}+\left(k_{1}-a_{n}\right) x_{1}- \\
-x_{2}^{4}+k_{2}^{\prime} x_{2}^{2}+\left(k_{2}-a_{n-1}\right) x_{2}-\ldots-x_{n}^{4}+ \\
+k_{n}^{\prime} x_{n}^{2}+\left(k_{n}-a_{1}\right) x_{n} . \\
y=x_{1} .
\end{array}\right.
$$


Then one of the steady states of the system is given by

$$
x_{1 s}=x_{2 s}=\ldots=x_{n s}=0 \text {. }
$$

Other steady states of the system (3) will be determined by solving the equation

$$
\begin{aligned}
& -x_{i s}^{3}-k_{i}^{\prime} x_{i s}+\left(k_{i}-a_{n-i+1}\right)=0, i=1, \ldots, \\
& x_{i s}^{2}=2 \sqrt[3]{\frac{a_{n-i+1}-k_{i}}{2}}, x_{j s}=0, i \neq j, i=1, \ldots, n, \\
& x_{i s}^{3,4}=\sqrt[3]{\frac{a_{n-1+i}-k_{i}}{2}}, x_{j s}=0, i \neq j, i=1, \ldots, n .
\end{aligned}
$$

\section{Stability of the steady states of the system}

We can investigate robust stability of the steady states (5) and (6) by using the basic provisions of the direct Lyapunov method [7, 8]. Asymptotic stability of the equilibrium points of the system can be shown if and only if there exists a positive definite Lyapunov function $V(x)$ such that its time derivative

$\dot{V}(x)$ along the solutions of the differential state equation is a negative definite function.

In our case, the time derivative of the Lyapunov function, is defined geometrically as the scalar product of the vector functions representing gradient of Lyapunov $(\partial V(x) / \partial x)$ and the velocity vector $(d x / d t)$. The gradient vector of a scalar Lyapunov function is pointed in the direction of increase of the latter, i.e., from the origin in the direction of the highest growth of Lyapunov functions. It should also be noted that in studies of stability of the systems reported in $[7,8]$ the origin corresponds to a given action or sets the state of the system. State equations (1) or (3) are always in deviations $\Delta x$ from the steady state $X_{S}\left(x=\Delta x=X-X_{S}\right)$. Therefore, the state equation (1) or (3) expresses the rate of change of a vector of deviations and we can assume that the velocity vector in a stable system is directed to the origin.

If the Lyapunov function $V(x)$ is specified as a vector function of $\left(V_{1}(x), V_{2}(x), \ldots, V_{n}(x)\right)$, for geometric interpretation of the components we choose antigradient Lyapunov functions $\left(-\partial V_{i}(x) / \partial x, i=1, \ldots, n\right)$ to be equal to the largest component of the velocity vector $(d x / d t)$ :

$$
-\frac{d x_{i}}{d t}=\frac{\partial V_{i}^{(x)}}{\partial x_{1}}+\frac{\partial V_{i}(x)}{\partial x_{2}}+\ldots+\frac{\partial V_{i}(x)}{\partial X_{n}}, i=1, \ldots, n
$$

Then the time derivative of the components of the vector Lyapunov function for stability of the steady state (4) will be:

$$
\left\{\begin{array}{l}
\frac{d V_{1}(x)}{d t}=-x_{2}^{2} \\
\frac{d V_{1}(x)}{d t}=-x_{3}^{2} \\
\cdots \frac{d V_{n-1}(x)}{d t}=-x_{n}^{2} \\
\frac{d V_{n}(x)}{d t}=\left[-x_{1}^{4}+k_{1}^{\prime} x_{1}^{2}+\left(k_{1}-a_{n}\right) x_{1}-x_{2}^{4}+k_{2}^{\prime} x_{2}^{2}+\right. \\
\left.+\left(k_{2}-a_{n-1}\right) x_{2}-, \ldots,-x_{n}^{4}+k_{n}^{\prime} x_{n}^{2}+\left(k_{n}-a_{1}\right) x_{n}\right]^{2} .
\end{array}\right.
$$

From the above, it follows that the total time derivative of the components of the vector function Lyapunov will always be a negative-definite function.

Also, the total time derivative of the Lyapunov function can be represented as a scalar function as follows

$$
\begin{aligned}
& \frac{d V(x)}{d t}=-x_{2}^{2}-x_{3}^{2}-\ldots-x_{n}^{2}- \\
& -\left[-x_{2}^{4}+k_{1}^{\prime} x_{1}^{2}+\left(k_{1}-a_{n}\right) x_{1}-x_{2}^{4}+k_{2}^{\prime} x_{2}^{2}+\right. \\
& \left.+\left(k_{2}-a_{n-1}\right) x_{2}-, \ldots,-x_{n}^{4}+k_{n}^{\prime} x_{n}^{2}+\left(k_{n}-a_{1}\right) x_{n}\right]^{2}
\end{aligned}
$$

It can be shown that the vector function of Lyapunov $\left(V_{i}, i=1, \ldots, n\right)$ shown by the components of the gradient vector is:

$$
\begin{aligned}
& \frac{\partial V_{1}(x)}{\partial x_{1}}=0, \frac{\partial V_{1}(x)}{\partial x_{2}}=-x_{2}, \frac{\partial V_{1}(x)}{\partial x_{3}}=0, \ldots, \frac{\partial V_{1}(x)}{\partial x_{n}}=0 \\
& \frac{\partial V_{2}(x)}{\partial x_{1}}=0, \frac{\partial V_{2}(x)}{\partial x_{2}}=0, \frac{\partial V_{2}(x)}{\partial x_{3}}=-x_{3}, \ldots, \frac{\partial V_{2}(x)}{\partial x_{n}}=0 \\
& \ldots \\
& \ldots \\
& \frac{\partial V_{n-1}(x)}{\partial x_{1}}=0, \frac{\partial V_{n-1}(x)}{\partial x_{2}}=0, \frac{\partial V_{n-1}(x)}{\partial x_{3}}=0, \frac{\partial V_{n-1}(x)}{\partial x_{n}}=-x_{n} \\
& -\frac{\partial V_{n}(x)}{\partial x_{1}}=-x_{1}^{4}+k_{1}^{\prime} x_{1}^{2}+\left(k_{1}-a_{n}\right) x_{1}, \\
& -\frac{\partial V_{n}(x)}{\partial x_{2}}=-x_{2}^{4}+k_{2}^{\prime} x_{2}^{2}+\left(k_{2}-a_{n-1}\right) x_{2}, \ldots, \\
& -\frac{\partial V_{n}(x)}{\partial x_{n}}=-x_{n}^{4}+k_{n}^{\prime} x_{n}^{2}+\left(k_{n}-a_{1}\right) x_{n} .
\end{aligned}
$$

Lyapunov function in the scalar form is found as: 


$$
\begin{aligned}
& V(x)=\frac{1}{5} x_{1}^{5}-\frac{1}{3} k_{1}^{\prime} x_{1}^{3}-\frac{1}{2}\left(k_{1}-a_{n}\right) x_{1}^{2}+\frac{1}{5} x_{2}^{5}- \\
& -\frac{1}{3} k_{2}^{\prime} x_{2}^{3}-\frac{1}{2}\left(k_{2}-a_{n-1}-1\right) x_{2}^{2}+, \ldots,+\frac{1}{5} x_{n}^{5}-\frac{1}{3} k_{n}^{\prime} x_{n}^{3}- \\
& -\frac{1}{2}\left(k_{n}-a_{1}-1\right) x_{n}^{2}=\frac{1}{5} x_{1}^{5}-\frac{1}{3} k_{1}^{\prime} x_{1}^{3}-\frac{1}{2}\left(k_{1}-a_{n}\right) x_{1}^{2}+ \\
& +\frac{1}{5} x_{2}^{5}-\frac{1}{3} k_{2}^{\prime} x_{2}^{3}-\frac{1}{2}\left(k_{2}-a_{n-1}-1\right) x_{2}^{2}+, \ldots,+\frac{1}{5} x_{n}^{5}- \\
& -\frac{1}{3} k_{n}^{\prime} x_{n}^{3}-\frac{1}{2}\left(k_{n}-a_{1}-1\right) x_{n}^{2} .
\end{aligned}
$$

It is not obvious if function $\mathrm{V}$ (x) from (7) is positivedefinite or negative-definite, so we will make use of Morse lemma from catastrophe theory.

Let the system given by (3) be in a state of equilibrium (stable or sustainable) i.e. in the steady state, where the velocity vector $(d x / d t=0)$ is zero. Then, the gradient of the Lyapunov function $\nabla V(x)=0$ is zero, the stationary states of the system are found by Morse lemma $\operatorname{det} V_{i j} \neq 0$ to guarantee the existence of a smooth change of variables, such that the Lyapunov function (7) can be represented locally in a quadratic form. It follows that it is necessary to calculate the Hessian matrix for the stationary states (5).

Stability matrix (Hessian) [3,4] for the steady state (5) is defined as

$$
V_{i j}\left(x_{S}\right)=\left\|\left.\frac{\partial^{2} V(x)}{\partial x_{i} \partial x_{j}}\right|_{x_{S=0}}\right\|=\|\| \begin{array}{ccccc}
\mu_{1} & 0 & 0 & \ldots & 0 \\
0 & \mu_{2} & 0 & \ldots & 0 \\
0 & 0 & \mu_{3} & \ldots & 0 \\
\ldots & \ldots & \ldots & \ldots & \ldots \\
0 & 0 & 0 & \ldots & \mu_{m}
\end{array} \|,
$$

where

$$
\begin{aligned}
& \frac{\partial^{2} V(x)}{\partial x_{i} \partial x_{j}}=0 \text { at } i \neq j \\
& \mu_{1}=-\left(k_{1}-a_{n}\right) \\
& \mu_{2}=-\left(k_{2}-a_{n-1}-1\right) \\
& \mu_{3}=-\left(k_{3}-a_{n-2}-1\right) \\
& \ldots \ldots \ldots . . \\
& \mu_{n}=-\left(k_{n}-a_{1}-1\right)
\end{aligned}
$$

Lyapunov function (7) in the vicinity of the steady state (4) can be represented in a quadratic form

$$
\begin{aligned}
& V(x)=-\left(k_{1}-a_{n}\right) x_{1}^{2}-\left(k_{2}-a_{n-1}-1\right) x_{2}^{2}- \\
& -\left(k_{3}-a_{n-2}-1\right) x_{3}^{2}-, \cdots,-\left(k_{n}-a_{1}-1\right) x_{n}^{2},
\end{aligned}
$$

Then conditions of robust stability of the steady state (5) are defined by the system of inequalities:

$$
a_{n}>k_{1}, a_{n-1}>k_{2}-1, a_{n-2}>k_{3}-1, \ldots, a_{1}>k_{n}-1, .
$$

Stability of steady states (5) and (6) is based on Lyapunov functions and can be obtained from:

$$
\begin{aligned}
& a_{n}<k_{1}, a_{n-1}<k_{2}-\frac{1}{221}, \\
& a_{n-2}<k_{3}-\frac{1}{221}, \ldots, a_{n}<k_{n}-\frac{1}{221}, \\
& a_{n}<k_{1}, a_{n-1}<k_{2}-\frac{1}{20}, \\
& a_{n-2}<k_{3}-\frac{1}{20}, \ldots, a_{1}<k_{n}-\frac{1}{20},
\end{aligned}
$$

Steady states (5) and (6) cannot exist at the same time. Only one of them exists at any moment and it will always be stable. With the loss of this state, there is a new steady state, and it will also be stable. This makes it possible to build a control system that will be stable when these conditional states are (10) and (11)

\section{Conclusion}

The results obtained by designing control systems with increased potential of robust stability and dynamics, allow to assure safety and efficiency of control systems in engineering and technology during their construction and operation.

We also developed an approach to designing control systems with increased potential of robust stability for linear objects with uncertain parameters by using control laws in the two-parameter class of structurally stable maps. It was shown that the system is asymptotically stable and steady state can be reached for both negative and positive change of uncertain parameters of the control object. When uncertain parameters transition through zero, bifurcation occurs and new stable branches are created. At the same time, zero steady state loses stability. These steady states do not exist at the same time and it becomes possible to build a system that is stable for any change in uncertain parameters.

\section{References}

[1] Polyak B.T., Sherbakov P.S. "Robust stability and Control" - p.303, Moscow, Nauka, 2002

[2] GregoireNicolis, Ilya Prigogine. Exploring Complexity an Introduction. - New York, 1989.

[3] Poston G., Stuart I. "Catastrophe Theory and Its Applications", Moscow, Mir, 1980

[4] Gilmor R., "Applied theory of catastrophes", Moscow, Mir, 1981

[5] M. Beisenbi, "Methods to improve the capacity of robust stability control systems," p. 352, Astana, 2011.

[6] M. Beisenbi, "Models and methods of system analysis and management of deterministic chaos in the economy," p. 201, Astana, 2011.

[7] Barabashkin E.A. "Introduction to the theory of stability" p.267,Moscow, Nauka, 1967.

[8] Malkin I. G., "The theory of stability of motion" p.540, Moscow, Nauka, 1966. 